\title{
PATCH BASED SUPER-RESOLUTION OF MR SPECTROSCOPIC IMAGES
}

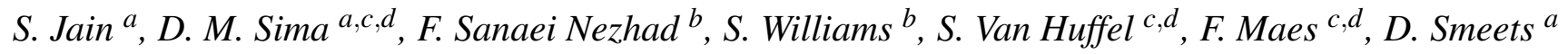 \\ $a$ icometrix, R\&D, Leuven, Belgium \\ ${ }^{b}$ Centre for Imaging Science, University of Manchester, Manchester, United Kingdom \\ ${ }^{c}$ Department of Electrical Engineering-ESAT, KU Leuven, Leuven, Belgium \\ ${ }^{d}$ iMinds, Medical IT, Leuven, Belgium
}

\begin{abstract}
In this paper, a new single-image super-resolution method is presented to increase the spatial resolution of metabolite maps computed from magnetic resonance spectroscopic imaging. The proposed method is based on a non-local patch-based strategy that uses a high resolution T1-weighted image to regularise the super-resolution process. The method is implemented in a multi-scale fashion. The accuracy of the method is validated on both phantom and in vivo images. Both qualitative and quantitative validation suggest that the method has potential for clinically relevant neuroimaging applications.
\end{abstract}

Index Terms - super-resolution, up-sampling, magnetic resonance spectroscopy imaging

\section{INTRODUCTION}

Magnetic resonance spectroscopy imaging (MRSI) of the brain provides chemical composition of tissue and may reveal underlying metabolic changes that are not visible on conventional magnetic resonance imaging (MRI) such as T1weighted MR imaging [1]. Despite the fact that MRSI provides complementary information w.r.t. conventional MRI, it is still not widely used in practice. A possible reason is that MRSI has a low signal to noise ratio, and thus requires large voxel sizes in order to capture sufficient signal. Typical voxel sizes in clinical MRSI protocols are around $1 \mathrm{~cm}^{3}$ [2]. High spatial resolution MRSI cannot be acquired in the clinical routine as it requires a long scanning time. For example, we observed that in order to acquire a brain MRS image with a PRESS sequence $(\mathrm{TR}=1500 \mathrm{~ms}, \mathrm{TE}=120$ $\mathrm{ms}, 160 \times 140 \mathrm{~mm}^{2}$ FOV, 4 axial slices, $5 \times 5 \times 5 \mathrm{~mm}^{3}$ voxel resolution, number of signal averages $=4$ ), approximately 40 minutes are required. Therefore, there is a need to create high resolution metabolic images from MRSI measurements with a short acquisition time using post-processing techniques. Super-resolution (SR) is a technique by which the spatial resolution of an image could be increased. Super-resolution methods can broadly be classified into two main groups. The first group of methods increases the spatial resolution during the acquisition, e.g., by increasing the k-space sampling
[3], or by acquiring multiple images. In case of multiple images, many low resolution images are either acquired from multiple directions or by shifting the voxel position by half of its size either in-plane [4] or in the slice direction [5]. Such methods requires a long acquisition time, and thus limit their use in clinical practice. The second group of methods relies on post-processing of single-image acquisition, where the SR process has to rely on additional regularity assumptions. These constraints could be imposed by incorporating information from other modalities, like tissue segmentations from MRI. This tissue information could be integrated with either k-space time MRSI signal [7] or with quantified MRSI metabolite map [9]. The latter strategy has less variables to be optimised, therefore, the SR model could be simplified.

The method proposed in this paper is a single-image SR method, which performs the up-sampling of quantified MRSI metabolite map from routinely acquired low-resolution MRSI data. The method is a patch-based technique [9], which is guided by the tissue segmentation of a high resolution T1weighted image. The proposed method is implemented in a multi-scale fashion. At each level, SR is performed in two steps. In the first step, the tissue segmentations are used in estimating the metabolite concentration in each higher resolution voxel. Then, in the second step, the estimated metabolite concentration in every high resolution voxel is corrected with the error towards the corresponding lower level voxel's metabolite value. To the best of our knowledge, a patch-based super-resolution technique has never been applied before for up-sampling MRSI-based metabolic images. We validate the method on an acetate (Ace) image from a phantom, which contains different Ace proportions in different spatial locations, as well as on $\mathrm{N}$-acetylaspartate (NAA) images from healthy volunteers.

\section{METHODS}

\subsection{Method description}

Let $y$ be the low resolution (assumed noise-free) metabolite map and $x$ be the unknown corresponding high resolution metabolite image defined over a high resolution voxel space 
$\Omega$. Theoretically,

$$
y=H(x)
$$

where $H$ is a known blur and downsample operator

In order to reconstruct $x$ given $y$, the reconstruction model could be formulated as an Euler Lagrange energy minimisation problem:

$$
\hat{x}=\arg \min \{\underbrace{\|y-H(x)\|^{2}}_{\text {least square fitting }}+\underbrace{\lambda R(x)}_{\text {regulariser term }}\}
$$

The $R(x)$ term preserves the geometry and regularity. Therefore, $R(x)$ could be defined as:

$$
R(x)=\sum_{i \in \Omega}\left\|x_{i}-E\left(x_{i} \mid \zeta_{i}\right)\right\|^{2}
$$

$E\left(x_{i} \mid \zeta_{i}\right)$ is the conditional reconstruction of $x_{i}$ based on voxel intensity values in its non-local neighbourhood $\zeta_{i}$. In this work, the reconstruction will benefit from the availability of a T1-weighted MRI along with its tissue segmentations; which are assumed to be co-registered to the low resolution MRS image. The pseudocode of the super-resolution method for solving (2) could be written as:

\section{Algorithm 2.1: $\operatorname{SR}(y=$ low res image; $x=$ starting value for high res image) \\ while no convergence \\ $\left\{\begin{array}{l}\text { Estimate } R(x) \\ U \text { pdate } x \text { by minimising }\|y-H(x)\|^{2}+\lambda R(x)\end{array}\right.$}

We assume that the term $E\left(x_{i} \mid \zeta_{i}\right)$ is consistent under strictly stationary conditions, i.e., this stationary condition amounts to saying that as the size of the image increases, we could find in the image many similar patches for all the details of the image. Also, as the distance between regions increases in the image, they will become more independent. This means when the regions move far from each other, their correlation is decreased [10]. Finally, the metabolite concentrations within a tissue type are more similar than with another tissue type. All of these assumptions are approximately met in our case. If $E\left(x_{i} \mid \zeta_{i}\right)$ is modelled under these assumptions, it could be proved [11] that $x_{i} \rightarrow E\left(x_{i} \mid \zeta_{i}\right)$ which means $R(x) \rightarrow 0$. Therefore, the two terms of the energy functional could be decoupled and asymptotically approximated as the reconstruction and mean correction steps of the new iterative super-resolution algorithm 2.2.

Algorithm 2.2: $\operatorname{SR}(y=$ low res image; $x=$ starting value for high res image)

$$
\begin{aligned}
& \text { while }\left|x_{i}^{t}-x_{i}^{t-1}\right|<\varepsilon, \forall x_{i} \in x \\
& \left\{\begin{array}{l}
\text { Reconstruction }: x_{i}^{t+1}=E\left(x_{i} \mid \zeta_{i}\right), \forall x_{i} \in x \\
\text { Mean correction }: \hat{x}_{i}^{t+1}=x_{i}^{t+1}-(\underbrace{\left(\left(_{L}^{1} \sum_{l=0}^{L} x_{l}^{t+1}\right)-y_{p}\right.}_{\text {error }}) .
\end{array}\right.
\end{aligned}
$$

In the mean correction step of algorithm 2.2, the error term imposes that the average of the $L$ reconstructed values $x_{l}$ composing the low resolution voxel $y_{p}$ (of which $x_{i}$ is part) must be equal to the corresponding original value of the low resolution image. This corresponds to a boxcar operator for $H$ in (1), but it could be easily replaced by a general smoothing and downsampling operator $H$.

The reconstruction term, $E\left(x_{i} \mid \zeta_{i}\right)$ is defined as:

$$
E\left(x_{i} \mid \zeta_{i}\right)=\sum_{j \in \zeta_{i}} w\left(x_{i}, x_{j}\right) * x_{j}
$$

where the weight $w\left(x_{i}, x_{j}\right)$ defines the contribution of neighbourhood voxel $x_{j}$ in the reconstruction of $x_{i}$. More similar neighbour means more weight to that neighbour. Similar neighbours are defined on the basis of tissue segmentation from MRI that were down-sampled to match the MRSI resolution. Mathematically, $w\left(x_{i}, x_{j}\right)$ could be defined as [8]:

$$
\begin{aligned}
& \qquad w\left(x_{i}, x_{j}\right)=\frac{1}{Z_{i}} e^{-\frac{1}{2 N}\left(\frac{\left\|\hat{N}_{i}-\hat{N}_{j}\right\|}{h_{i}}\right)^{2}} \\
& \text { where } Z_{i} \text { is a normalisation constant such that } \sum_{j \in \zeta_{i}} w\left(x_{i}, x_{j}\right)=1,
\end{aligned}
$$

$\hat{N}_{i}$ is the intensity vector of the local neighbourhood $N_{i}$ of length $N$,

$h$ is the standard deviation of neighbourhood voxel intensities.

In our case, the local neighbourhood parameter $\left(N_{i}\right)$ is empirically defined as a cube of size 8 voxels around the centre voxel $x_{i}$. The parameter $h$ is the standard deviation of intensities in this cube, computed only among voxels with similar tissue composition as $x_{i}$. Since we use both MRI and MRSI, $E\left(x_{i} \mid \zeta_{i}\right)$ in its generalised form could be defined similar to [9]:

$$
E\left(x_{i} \mid \zeta_{i}\right)=\sum_{j \in \zeta_{i}}\left\{\left(1-\alpha\left(x_{i}\right)\right) * w_{M R I}+\alpha\left(x_{i}\right) * w_{M R S I}\right\} * x_{j}
$$

where $\alpha\left(x_{i}\right)$ is a weighing term between the weights of the MRI and MRSI. In the current study, the reconstruction process is driven by MRI only, and therefore, $\alpha\left(x_{i}\right)$ is chosen to be zero. Of course, if the application requires incorporation of prior knowledge from MRSI, $\alpha\left(x_{i}\right)$ could be chosen accordingly.

The method is implemented in a multi-scale fashion to incorporate different MR image features at each scale for the reconstruction of the metabolite image. Starting from the low resolution metabolite image, each iteration increases resolution by a certain factor (e.g., by a factor of 2) in each spatial direction, until the desired super-resolution (e.g., a resolution close to the one of the conventional MRI) is reached.

\subsection{Data}

\subsubsection{Phantom data}

A cylindrical phantom (180 mm diameter, $168 \mathrm{~mm}$ depth) is used, consisting of 7 small cylinders each having a diameter 
of $40 \mathrm{~mm}$ and depth of $150 \mathrm{~mm}$, going through the thickness of the phantom as shown in figure 1. The first cylinder was kept empty for orientation purposes, followed by the second cylinder having Ace concentration of 6 units. Then, the Ace concentration was increased by 2 units for the next cylinder resulting in a concentration of 16 units for the last cylinder. The rest of the empty space in the phantom was filled with water. For comparison purpose, we are interested in cylinder 5 and 7 that have twice the Ace concentration of cylinder 2 and 3 respectively (figure 1). MR imaging was performed on a 3T whole body scanner (Philips Medical Systems Achieva). The protocol contained two sequences: 3D T1weighted fast field echo (FFE) sequence (TR $6.6 \mathrm{~ms}$, TE 3.12 ms, FA $9^{\circ}, 180 \times 180 \mathrm{~mm}^{2}$ FOV, 3 axial slices, $0.8 \times 0.8 \times 10.0$ $\mathrm{mm}^{3}$ voxel resolution) and the ${ }^{1} \mathrm{H}$ MRSI PRESS sequence $\left(\mathrm{TR}=1500 \mathrm{~ms}, \mathrm{TE}=120 \mathrm{~ms}, 300 \times 300 \mathrm{~mm}^{2}\right.$ FOV, $3 \mathrm{ax}-$ ial slice, $10.0 \times 10.0 \times 10.0 \mathrm{~mm}^{3}$ voxel resolution). MRI individual cylinder segmentation was performed manually using Slicer tool (version 4.3.1) [12] and were down-sampled to match the MRSI resolution at each scale. MRSI data was quantified using the SPID software [13] to obtain metabolite images for Ace.

\subsubsection{In-vivo data}

Six healthy volunteers ( 2 males, 4 females, age 26-29) participated in a study at NIHR/WT Clinical Research Centre, Central Manchester NHS Foundation Trust, Manchester, UK. The study was approved by the local ethics committee and all volunteers signed informed consent forms. MR imaging was performed on a 3T whole body scanner (Philips Medical Systems Achieva). The protocol contained two sequences: 3D T1weighted fast field echo (FFE) sequence (TR $8.6 \mathrm{~ms}$, TE $4 \mathrm{~ms}$, FA $8^{\circ}, 256 \times 256 \mathrm{~mm}^{2}$ FOV, 140 axial slices, $1.0 \times 1.0 \times 1.0$ $\mathrm{mm}^{3}$ voxel resolution) and low resolution ${ }^{1} \mathrm{H}$ MRSI PRESS sequence $\left(\mathrm{TR}=1500 \mathrm{~ms}, \mathrm{TE}=120 \mathrm{~ms}, 160 \times 140 \mathrm{~mm}^{2} \mathrm{FOV}\right.$, 2 axial slices, $10.0 \times 10.0 \times 10.0 \mathrm{~mm}^{3}$ voxel resolution, number of signal averages $=4$ ) and high resolution ${ }^{1} \mathrm{H}$ MRSI PRESS sequence $(\mathrm{TR}=1500 \mathrm{~ms}, \mathrm{TE}=120 \mathrm{~ms}, 160 \times 140$ $\mathrm{mm}^{2}$ FOV, 4 axial slices, $5.0 \times 5.0 \times 5.0 \mathrm{~mm}^{3}$ voxel resolution, number of signal averages $=4$ ). MRI tissue segmentation was performed using MSmetrix [14] and were down-sampled to match the MRSI resolution at each scale. MRSI data was quantified using the SPID software to obtain metabolite images for NAA.

\section{RESULTS}

\subsection{Accuracy test on phantom data}

Table 1 presents the quantitative results of Ace concentration ratio between cylinder 5 and cylinder 2 both at low resolution (acquired at $10 \times 10 \times 10 \mathrm{~mm}^{3}$ ) and at high resolution (reconstructed at $1.25 \times 1.25 \times 1.25 \mathrm{~mm}^{3}$ ). Since cylinder 5 that has twice the Ace concentration of cylinder 2, this relation holds better at the high resolution. We also observe a better relation between cylinder 7 and cylinder 3 at the high resolution.

Table 1: Comparison between Ace concentration at low resolution $\left(10 \times 10 \times 10 \mathrm{~mm}^{3}\right)$ and high resolution $\left(1.25 \times 1.25 \times 1.25 \mathrm{~mm}^{3}\right)$.

\begin{tabular}{|c|c|c|}
\hline Ace ratio (median) & $\frac{\text { cylinder } 5}{\text { cylinder } 2}$ & $\frac{\text { cylinder } 7}{\text { cylinder } 3}$ \\
\hline Low resolution & 2.33 & 1.80 \\
\hline High resolution & 1.86 & 1.89 \\
\hline
\end{tabular}

In order to compare the results in a qualitative manner, figure 1 presents the Ace maps acquired at low resolution and reconstructed at high resolution. It could be seen that at high resolution, by incorporating the tissue segmentation information, we reduced the partial volume effects considerably.

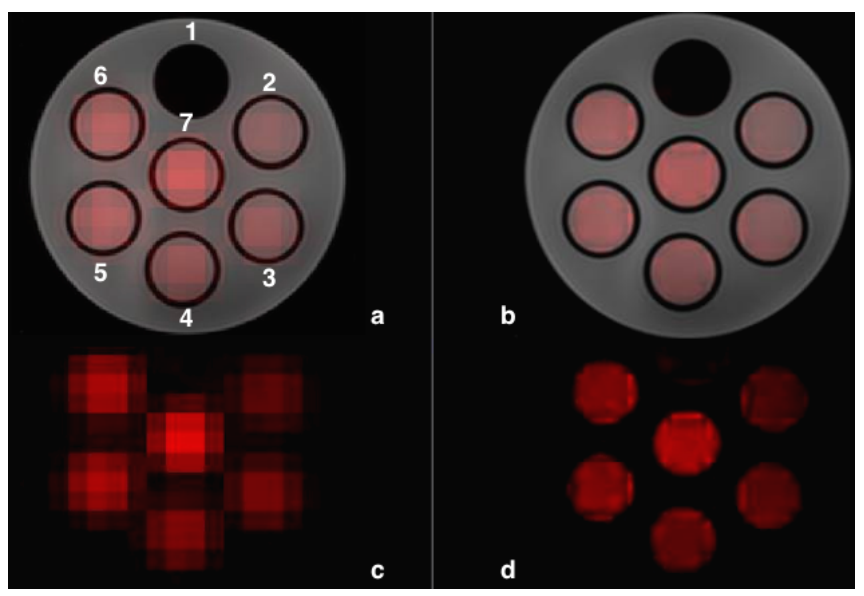

Fig. 1: High resolution Ace map reconstruction using the proposed method. (a) T1-weighted image overlaid with acquired low resolution Ace map, (b) T1-weighted image overlaid with reconstructed high resolution Ace map, (c) Acquired low resolution Ace map, (d) Reconstructed high resolution Ace map.

\subsection{Accuracy test on in-vivo data}

Table 2 presents the quantitative results of NAA concentration ratio between gray matter (GM) and white matter (WM) at the acquired low resolution, ALR $\left(10 \times 10 \times 10 \mathrm{~mm}^{3}\right)$, at the acquired high resolution, AHR $\left(5 \times 5 \times 5 \mathrm{~mm}^{3}\right)$ and reconstructed NAA concentration at high resolution (RHR) for all volunteers. A slightly high median value of GM to WM NAA ratio in the RHR compared to the ALR is because of reduced partial volume effects at high resolution, which is desirable. AHR reflects a higher NAA concentration ratio between GM and WM, but we did not confirm the trend that the (global) NAA concentration is higher in GM compared to WM, as reported in some studies $[15,16]$. 
Table 2: Comparison of NAA concentration ratio between gray matter (GM) and white matter (WM) at the acquired low resolution $(\mathrm{ALR})\left(10 \times 10 \times 10 \mathrm{~mm}^{3}\right)$, at the acquired high resolution (AHR) $\left(5 \times 5 \times 5 \mathrm{~mm}^{3}\right)$ and reconstructed NAA concentration at high resolution (RHR).

\begin{tabular}{lccc}
\hline & \multicolumn{3}{c}{$N A A_{G M}: N A A_{W M}$ (median) } \\
& ALR & AHR & RHR \\
\hline Subject-1 & 0.81 & 0.82 & 0.84 \\
\hline Subject-2 & 0.82 & 0.83 & 0.86 \\
\hline Subject-3 & 0.87 & 0.98 & 0.88 \\
\hline Subject-4 & 0.98 & 1.0 & 1.0 \\
\hline Subject-5 & 0.87 & 0.98 & 0.85 \\
\hline Subject-6 & 0.73 & 0.81 & 0.76 \\
\hline
\end{tabular}

In order to compare the results in a qualitative manner, figure 2 presents the NAA maps at the acquired low resolution, high resolution and reconstructed NAA map at high resolution for subject-1. It could be seen that the reconstructed NAA map is smoother than the NAA map at the acquired high resolution. Thus, the effect of noise is reduced. Moreover, due to the incorporation of tissue segmentation, the reduction in partial volume could been seen at the inter-hemispheric fissure boundary. On the other hand, it seems that the correlation between the reconstructed NAA map and the acquired NAA map at high resolution is rather low and requires further investigation.

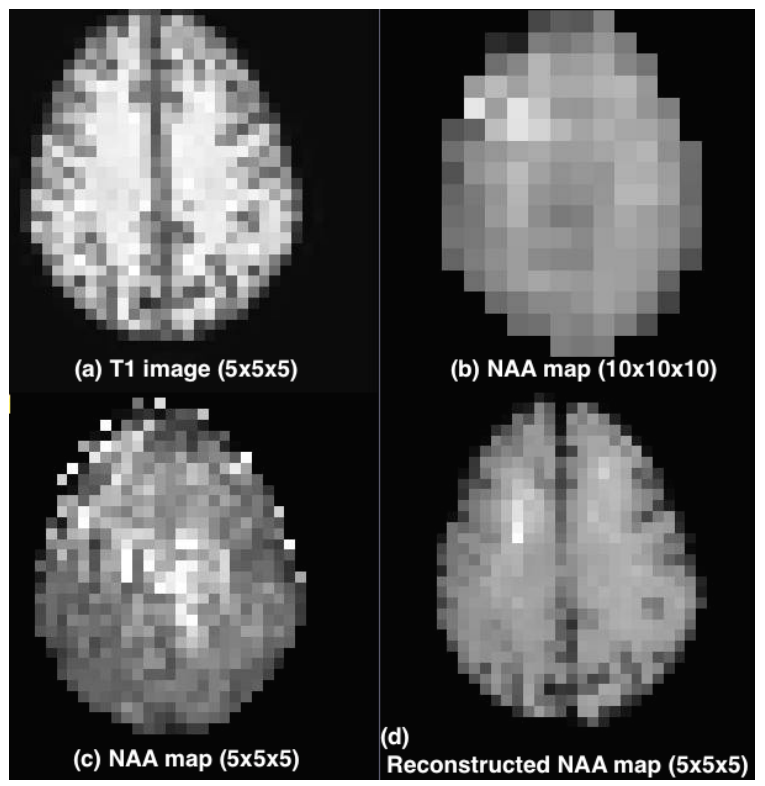

Fig. 2: High resolution NAA map reconstruction using the method for subject-1. The T1-weighted image (a) is resampled to the NAA space. The NAA maps shown in (b) and (c) are quantified at the spatial resolution at which they were acquired.

\section{DISCUSSION AND CONCLUSION}

High resolution metabolite images of the brain are important in clinical applications, where variations in metabolite content might occur at sub-voxel level and thus might not be visible at macroscopic resolution. Indeed, some studies have shown that metabolite content at low resolution does not reveal significant differences between metabolite levels in pathologic or normal tissue; only after partial volume correction using the CSF proportion per voxel, these differences became significant [17].

Methods for achieving SR of MRSI often require prolonged acquisition time, which is unfeasible in clinical routine. Nevertheless, single-image SR has been shown to be feasible in other neuroimaging applications where the image of interest has lower resolution compared to conventional MRI (PET, DWI). Conventional MRI could serve as regulariser and guide the SR algorithm towards similarities and differences between various patches in the high resolution reconstruction.

In this work, we propose a simple patch-based superresolution scheme that iterates between an up-sampled reconstruction step and a mean correction step, until convergence. The reconstruction step relies on regularity assumptions derived from image segmentation and amounts to the fact that image patches having a similar tissue composition should influence each other's higher resolution reconstruction. The mean correction step keeps the reconstruction under control by requiring that the reconstructed higher resolution image is consistent with the underlying low resolution metabolite image. It is important to note that the method could not be expected to recover small scale features that were not sufficiently picked up by the low resolution measurement.

We validated the method on Ace images quantified from a phantom and NAA from brain images of healthy volunteers. Initial validation suggests that the method has potential and therefore, as a future step, we intend to further validate the technique on more healthy volunteers as well as in pathological neurological conditions such as multiple sclerosis and psychiatric illnesses.

\section{ACKNOWLEDGEMENTS}

This research received funding from the TRANSACT (EUFP7-PEOPLE-2012-ITN-316679); EPSRC, and the research support from the NIHR/WT Clinical Research Facility.

\section{REFERENCES}

[1] Á. Rovira, C. Auger, and J. Alonso, "Magnetic resonance monitoring of lesion evolution in multiple sclerosis," Therapeutic Advances in Neurological Disorders, vol. 6, pp. $298-310,2013$. 
[2] S. J. Nelson, "Magnetic resonance spectroscopic imaging. evaluating responses to therapy for gliomas," IEEE engineering in medicine and biology magazine : the quarterly magazine of the Engineering in Medicine \& Biology Society, vol. 23, pp. 30-39, 2004.

[3] S. W. Chung, Y. Lu, and R. G. Henry, "Comparison of bootstrap approaches for estimation of uncertainties of DTI parameters," NeuroImage, vol. 33, pp. 531-541, 2006.

[4] S. Peled and Y. Yeshurun, "Superresolution in MRI: application to human white matter fiber tract visualization by diffusion tensor imaging," Magnetic Resonance in Medicine, vol. 45, pp. 29-35, 2001.

[5] H. Greenspan, G. Oz, N. Kiryati, and S. Peled, "MRI inter-slice reconstruction using super-resolution," Magnetic Resonance Imaging, vol. 20, pp. 437 - 446, 2002.

[6] X. Hu et al., "Slim: Spectral localization by imaging," Magnetic Resonance in Medicine, vol. 8, pp. 314-322, 1988.

[7] J. Kornak et al., "Bayesian k-space time reconstruction of MR spectroscopic imaging for enhanced resolution," IEEE transaction in Medical imaging, vol. 29, pp. 1330-13350, 2010.

[8] P. Coupé, J.V. Manjón, M. Chamberland, M. Descoteaux, and B. Hiba, "Collaborative patch-based super-resolution for diffusion-weighted images," NeuroImage, vol. 83, pp. 245 - 261, 2013.

[9] F. Rousseau and The Alzheimer's Disease Neuroimaging Initiative, "A non-local approach for image superresolution using intermodality priors," Medical Image Analysis, vol. 14, pp. $594-605,2010$.

[10] A. Buades, B. Coll, and J. M. Morel, "A review of image denoising algorithms, with a new one," Simul, vol. 4, pp. 490-530, 2005.

[11] G. G. Roussas, "Nonparametric regression estimation under mixing conditions," Stochastic Processes and their Applications, vol. 36, pp. 107 - 116, 1990.

[12] 3D Slicer, "www.slicer.org," Online; accessed 20 Oct. 2015.

[13] SPID, "http://homes.esat.kuleuven.be/ biomed/software.php," Online; accessed 20 Oct. 2015.

[14] S. Jain et al., "Automatic segmentation and volumetry of multiple sclerosis brain lesions from MR images," NeuroImage: Clinical, vol. 8, pp. 367 - 375, 2015.
[15] Y. Wang and S.Li, "Differentiation of metabolic concentrations between gray matter and white matter of human brain by in vivo ${ }^{1} \mathrm{H}$ magnetic resonance spectroscopy," Magnetic Resonance in Medicine, vol. 39, pp. 28-33, 1998.

[16] M. Inglese et al., "Global average gray and white matter N-acetylaspartate concentration in the human brain," NeuroImage, vol. 41, pp. 270 - 276, 2008.

[17] W. Weber-Fahr et al., "A fully automated method for tissue segmentation and CSF-correction of proton MRSI metabolites corroborates abnormal hippocampal NAA in schizophrenia," NeuroImage, vol. 16, pp. $49-60$, 2002. 